\title{
An update on the central nervous system manifestations of familial tumor predisposition syndromes
}

\author{
David A. Solomon ${ }^{1}[$ \\ Received: 26 January 2020 / Accepted: 26 January 2020 / Published online: 3 February 2020 \\ c) Springer-Verlag GmbH Germany, part of Springer Nature 2020
}

As we now know, cancer is the result of the combination of inherited genetic alterations and acquired somatic alterations over time, either resulting from spontaneous errors during DNA replication or in response to exogenous genotoxic stress such as ultraviolet irradiation or tobacco smoke. The combination of these inherited and acquired alterations cause activation of oncogenes and inactivation of tumor suppressor genes that result in tumorigenesis. While many cancers are "sporadic" being caused predominantly by acquired somatic alterations, a substantial fraction of cancers, particularly those arising during childhood, are "familial" and arise due to an underlying tumor predisposition syndrome caused by an inherited germline mutation. These germline mutations can be either inherited across multiple generations or can arise de novo in the sperm or the egg giving rise to the affected proband. Alternatively, tumor predisposition syndromes can also arise de novo due to constitutional mosaicism, in which a mutation is acquired during post-zygotic development in utero and is only present in a subset of the cells in an affected individual.

Most familial tumor predisposition syndromes have an autosomal dominant inheritance pattern, which results from an inherited germline mutation or deletion involving one of two alleles of a tumor suppressor gene. Neoplasms then develop in affected individuals when somatic inactivation of the remaining wild type allele occurs during the patient's lifetime, frequently during childhood or early adulthood. This second hit is most often a loss of heterozygosity event due to chromosomal nondisjunction during cell division, but can also occur due to somatic acquisition of a second mutation or deletion occurring in trans with the germline

David A. Solomon

david.solomon@ucsf.edu

1 Division of Neuropathology, Department of Pathology and Helen Diller Family Comprehensive Cancer Center, University of California, San Francisco, 513 Parnassus Ave, Health Sciences West 451, San Francisco, CA 94143, USA alteration. Some common examples of autosomal dominant tumor predisposition syndromes are Li-Fraumeni syndrome, Lynch syndrome, and neurofibromatosis type 1 (NF1) and type 2 (NF2). In contrast, some familial tumor predisposition syndromes have an autosomal recessive inheritance pattern, which results from either homozygous or compound heterozygous germline mutations causing biallelic inactivation of a tumor suppressor gene. The cancers that arise in autosomal recessive tumor predisposition syndromes almost always occur during early childhood. Examples of autosomal recessive tumor predisposition syndromes are constitutional mismatch repair deficiency syndrome (CMMRD) and Fanconi anemia.

It is now appreciated that the nervous system is affected in many familial tumor predisposition syndromes and that a substantial fraction of nervous system tumors results from one of the many different tumor predisposition syndromes that have been described to date. Recent genome sequencing studies have demonstrated that approximately $8 \%$ of all childhood malignancies arise in patients with pathogenic germline alterations causative of a known tumor predisposition syndrome [10, 21]. Other studies have suggested that this percentage may be even higher in children with brain tumors in particular [12]. Certain tumors of the central nervous system (CNS) are now known to be highly enriched in patients with tumor predisposition syndromes. For example, more than half of atypical teratoid/rhabdoid tumors occurring in infants under 1 year of age arise due to a germline mutation in the Swi/Snf chromatin remodeling gene SMARCB1 as part of rhabdoid tumor predisposition syndrome type 1 [3]. Approximately half of optic pathway gliomas arise in the setting of NF1 due to a germline mutation in the NFl tumor suppressor gene [15]. Multiple studies have now demonstrated that medulloblastoma is a disease that results from an inherited tumor syndrome in approximately $10 \%$ of children, which can be due to several different syndromes including Li-Fraumeni, Gorlin, and Fanconi anemia [4, 20]. In particular, the SHH-activated molecular 
subgroup of medulloblastomas has the highest contribution of inherited germline predisposition in up to $20 \%$ of affected children.

As such, a detailed family history of cancer, personal history of neoplasia, and physical examination evaluating for manifestations of a tumor predisposition syndrome (e.g. Lisch nodules, neurofibromas, café-au-lait macules, shagreen patch, etc.) should be performed for all patients with CNS tumors. Notably, not all tumor predisposition syndromes have cutaneous manifestations (e.g. Li-Fraumeni syndrome), and the absence of a family history of cancer does not exclude the presence of a tumor predisposition syndrome which has arisen due to an acquired de novo germline mutation. Genetic counseling and germline sequencing should be routinely considered in all children with CNS tumors, and also in those adults with CNS tumors in which there is clinical suspicion for a tumor predisposition syndrome based on family history, physical examination, or other factors.

Diagnosis of a familial tumor predisposition syndrome has important implications both for the patient as well as any family members that are also affected. Such recognition allows for appropriate surveillance and screening following recommended guidelines such as the modified "Toronto protocol" that has been established for individuals with Li-Fraumeni syndrome [13]. This can lead to early detection and potentially life-saving intervention. Additionally, recognition that a cancer is arising as part of a tumor predisposition syndrome may have implications for immediate treatment purposes. For instance, current recommendations are to avoid radiation therapy if possible in individuals with Li-Fraumeni syndrome given the high risk of inducing sarcomas and other neoplasms due to the DNA double-strand breaks caused by the gamma-radiation in cells haploinsufficient for functional p53 protein [16]. Additionally, the hypermutation in glioblastomas resulting from mismatch repair deficiency due to either Lynch syndrome or CMMRD may result in increased efficacy of immune checkpoint inhibitors [2,14].

Investigation into the biology and genetics of tumors arising in the setting of familial tumor predisposition syndromes has revealed similarities to their sporadic counterparts in some cases, but that some syndrome-associated tumors may be distinct from their sporadic counterparts. For example, approximately half of choroid plexus carcinomas arise in the setting of Li-Fraumeni syndrome due to germline TP53 mutation [16]. However, the majority of sporadic choroid plexus carcinomas lack somatic TP53 mutations and have favorable outcome compared to TP53 mutant choroid plexus carcinomas [19]. Thus, Li-Fraumeni syndrome-associated choroid plexus carcinomas appear to be biologically distinct from their sporadic counterparts in most cases. Most pilocytic astrocytomas are sporadic and arise due to somatic $B R A F$ fusion or mutation. However, the clinical scenario of a pilocytic astrocytoma that lacks $B R A F$ fusion or mutation should raise suspicion for either NF1 or Noonan syndrome, which account for a substantial subset of $B R A F$ wild type pilocytic astrocytomas, particularly those involving the optic pathway. Gliomas arising in the setting of NF1 appear to be distinct from their sporadic counterparts not only in terms of the genetic driver, but also histomorphology and clinical outcomes [15]. As such, it may be prudent for pathologists to specifically designate syndromic tumors as part of the integrated diagnostic framework recommended by the WHO Classification of Tumors of the Central Nervous System. For example, "Pilocytic astrocytoma, WHO grade I, arising in the setting of neurofibromatosis type 1" is more diagnostically informative than just "Pilocytic astrocytoma, WHO grade I", as it provides information of arising in association with germline NF1 inactivation and not the somatic BRAF fusion or mutation that is typical of sporadic tumors.

Occasionally, we encounter patients with multiple tumors or a strong family history of cancer who do not fit into the tumor spectrum associated with the known familial tumor predisposition syndromes and are not identified to harbor a pathogenic germline mutation in one of the associated genes. Efforts are currently underway to better understand the known familial tumor syndromes, and also to identify new tumor associations and novel tumor predisposition syndromes. For example, the Gliogene consortium has been investigating familial glioma and recently identified that germline mutation in the shelterin complex gene POTI causes a familial form of oligodendroglioma [1]. Additional studies have recently shown that ependymomas can arise as part of multiple endocrine neoplasia syndrome type 1 due to germline MEN1 mutation [8], and that both pleomorphic xanthoastrocytoma and diffuse astrocytoma can arise as part of familial melanoma-astrocytoma syndrome due to germline $C D K N 2 A$ deletion [5]. Beyond the longstanding link between germline NF2 mutation and meningiomas, recent studies have shown that meningiomas with rhabdoid or papillary morphology can arise as part of the $B A P 1$ tumor predisposition syndrome [17] and that clear cell meningiomas often arise due to germline SMARCE1 mutation [18]. Future investigation will identify the complete spectrum and incidence of CNS tumors that arise as part of familial tumor predisposition syndromes and will likely reveal that these syndromic tumors are often distinct biological entities compared to their sporadic counterparts associated with divergent outcomes and requiring unique therapy regimens.

In this issue of Acta Neuropathologica, I have organized a cluster of reviews on a few of the most common familial tumor syndromes with central nervous system manifestations. These in-depth reviews provide the most up-to-date information on the estimated incidence, diagnostic criteria, clinical features, histopathology, molecular pathogenesis, surveillance recommendations, and specific treatments for 
affected patients. Jennifer Cotter has reviewed CNS manifestations of tuberous sclerosis including autism spectrum disorder, cortical tubers, and subependymal giant cell astrocytoma [6]. Fausto Rodriguez and colleagues have reviewed the manifestations of NF1 including peripheral nerve sheath tumors, optic pathway glioma, malignant astrocytoma, and vasculopathies [15]. Sandro Santagata and colleagues have reviewed the manifestations of NF2 including meningioma, schwannoma, and ependymoma [7]. Brent Orr and colleagues have reviewed the CNS manifestations of Li-Fraumeni syndrome including diffuse gliomas, medulloblastoma, and choroid plexus carcinoma [16]. Sanda Alexandrescu and colleagues have reviewed the manifestations of DICERI syndrome including pineoblastoma, pituitary blastoma, and primary intracranial sarcoma [9]. And lastly, Cynthia Hawkins and colleagues have reviewed the different brain tumor polyposis syndromes including Lynch syndrome and CMMRD [11]. I hope you enjoy and learn from this spectacular cluster of reviews authored by the leading experts in the field.

Acknowledgements D.A. Solomon is supported by the NIH Director's Early Independence Award from the Office of the Director, National Institutes of Health (DP5 OD021403), a Developmental Research Program Award from the UCSF Brain Tumor SPORE grant from the National Cancer Institute, National Institutes of Health (P50 CA097257), the UCSF Glioblastoma Precision Medicine Program from the Sandler Foundation, the UCSF Wolfe Meningioma Program, and the UCSF Physician-Scientist Scholar Program.

\section{Compliance with ethical standards}

Conflict of interest No potential conflicts of interest to disclose.

\section{References}

1. Bainbridge MN, Armstrong GN, Gramatges MM, Bertuch AA, Jhangiani SN, Doddapaneni H et al (2014) Germline mutations in shelterin complex genes are associated with familial glioma. J Natl Cancer Inst 107:384. https://doi.org/10.1093/jnci/dju384

2. Bouffet E, Larouche V, Campbell BB, Merico D, de Borja $\mathrm{R}$, Aronson $\mathrm{M}$ et al (2016) Immune checkpoint inhibition for hypermutant glioblastoma multiforme resulting from germline biallelic mismatch repair deficiency. J Clin Oncol 34:22062211. https://doi.org/10.1200/JCO.2016.66.6552

3. Bourdeaut F, Lequin D, Brugières L, Reynaud S, Dufour C, Doz F et al (2011) Frequent hSNF5/INI1 germline mutations in patients with rhabdoid tumor. Clin Cancer Res 17:31-38. https ://doi.org/10.1158/1078-0432.CCR-10-1795

4. Brugières L, Remenieras A, Pierron G, Varlet P, Forget S, Byrde $V$ et al (2012) High frequency of germline SUFU mutations in children with desmoplastic/nodular medulloblastoma younger than 3 years of age. J Clin Oncol 30:2087-2093. https://doi. org/10.1200/JCO.2011.38.7258

5. Chan AK, Han SJ, Choy W, Beleford D, Aghi MK, Berger MS et al (2017) Familial melanoma-astrocytoma syndrome: synchronous diffuse astrocytoma and pleomorphic xanthoastrocytoma in a patient with germline CDKN2A/B deletion and a significant family history. Clin Neuropathol 36:213-221. https://doi.org/10.5414/NP301022

6. Cotter JA (2019) An update on the central nervous system manifestations of tuberous sclerosis complex. Acta Neuropathol. https://doi.org/10.1007/s00401-019-02003-1. (Epub ahead of print)

7. Coy S, Rashid R, StemmerRachamimov A, Santagata S (2019) An update on the CNS manifestations of neurofibromatosis type 2. Acta Neuropathol. https://doi.org/10.1007/s00401-019-02029 -5. (Epub ahead of print)

8. CuevasOcampo AK, Bollen AW, Goode B, Pajtler KW, Chavez L, Sharma T et al (2017) Genetic confirmation that ependymoma can arise as part of multiple endocrine neoplasia type 1 (MEN1) syndrome. Acta Neuropathol 133:661-663. https:// doi.org/10.1007/s00401-017-1689-7

9. de Kock L, Priest JR, Foulkes WD, Alexandrescu S (2019) An update on the central nervous system manifestations of DICER1 syndrome. Acta Neuropathol. https://doi.org/10.1007/s0040 1-019-01997-y. (Epub ahead of print)

10. Gröbner SN, Worst BC, Weischenfeldt J, Buchhalter I, Kleinheinz K, Rudneva VA et al (2018) The landscape of genomic alterations across childhood cancers. Nature 555:321-327. https ://doi.org/10.1038/nature25480

11. Kim B, Tabori U, Hawkins C (2020) An update on the CNS manifestations of brain tumor polyposis syndromes. Acta Neuropathol. https://doi.org/10.1007/s00401-020-02124-y. (Epub ahead of print)

12. Kline CN, Joseph NM, Grenert JP, van Ziffle J, Talevich E, Onodera $C$ et al (2017) Targeted next-generation sequencing of pediatric neuro-oncology patients improves diagnosis, identifies pathogenic germline mutations, and directs targeted therapy. Neuro Oncol 19:699-709. https://doi.org/10.1093/neuonc/ now 254

13. Kratz CP, Achatz MI, Brugières L, Frebourg T, Garber JE, Greer $\mathrm{MC}$ et al (2017) Cancer screening recommendations for individuals with Li-Fraumeni syndrome. Clin Cancer Res 23:e38-e45. https://doi.org/10.1158/1078-0432.CCR-17-0408

14. Le DT, Durham JN, Smith KN, Wang H, Bartlett BR, Aulakh LK et al (2017) Mismatch repair deficiency predicts response of solid tumors to PD-1 blockade. Science 357:409-413. https ://doi.org/10.1126/science.aan6733

15. Nix JS, Blakeley J, Rodriguez FJ (2019) An update on the central nervous system manifestations of neurofibromatosis type 1 . Acta Neuropathol. https://doi.org/10.1007/s00401-019-02002-2. (Epub ahead of print)

16. Orr BA, Clay MR, Pinto EM, Kesserwan C (2019) An update on the central nervous system manifestations of Li-Fraumeni syndrome. Acta Neuropathol. https://doi.org/10.1007/s0040 1-019-02055-3. (Epub ahead of print)

17. Shankar GM, Abedalthagafi M, Vaubel RA, Merrill PH, Nayyar N, Gill CM et al (2017) Germline and somatic BAP1 mutations in high-grade rhabdoid meningiomas. Neuro Oncol 19:535-545. https://doi.org/10.1093/neuonc/now235

18. Smith MJ, O'Sullivan J, Bhaskar SS, Hadfield KD, Poke G, Caird J et al (2013) Loss-of-function mutations in SMARCE1 cause an inherited disorder of multiple spinal meningiomas. Nat Genet 45:295-298. https://doi.org/10.1038/ng.2552

19. Tabori U, Shlien A, Baskin B, Levitt S, Ray P, Alon N et al (2010) TP53 alterations determine clinical subgroups and survival of patients with choroid plexus tumors. J Clin Oncol 28:1995-2001. https://doi.org/10.1200/JCO.2009.26.8169

20. Waszak SM, Northcott PA, Buchhalter I, Robinson GW, Sutter C, Groebner S et al (2018) Spectrum and prevalence of genetic predisposition in medulloblastoma: a retrospective genetic study and prospective validation in a clinical trial cohort. 
Lancet Oncol 19:785-798. https://doi.org/10.1016/S1470 -2045(18)30242-0

21. Zhang J, Walsh MF, Wu G, Edmonson MN, Gruber TA, Easton J et al (2015) Germline mutations in predisposition genes in pediatric cancer. N Engl J Med 373:2336-2346. https://doi.org/10.1056/ NEJMoa1508054
Publisher's Note Springer Nature remains neutral with regard to jurisdictional claims in published maps and institutional affiliations. 